\title{
Los hongos liquenizados del género Glyphis en Veracruz, México
}

\author{
The lichenized fungi of the genus Glyphis in \\ Veracruz, Mexico
}

\section{Acta Botanica Mexicana}

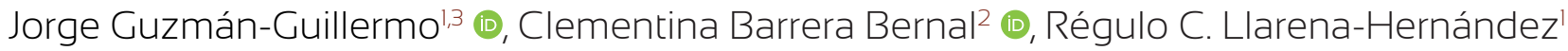

\section{Resumen}

Antecedentes y Objetivos: La familia Graphidaceae es considerada como una de las de mayor representatividad de hongos liquenizados, tanto en México como en el mundo. Aproximadamente 175 de sus especies se han registrado en México. El objetivo de este estudio fue realizar una revisión taxonómica de Glyphis en Veracruz, mediante el análisis de literatura y colectas de material proveniente de la zona tropical del gradiente altitudinal de "Cofre de Perote", Veracruz, México.

Métodos: Se colectaron microlíquenes de localidades ubicadas en ecosistemas tropicales del gradiente altitudinal de "Cofre de Perote", Veracruz, México, en bosque mesófilo de montaña y selva baja caducifolia. El material se procesó y depositó en el herbario XALU, y se analizó utilizando técnicas convencionales de liquenología respecto a morfología y macroquímica. Se consultaron bases de datos y trabajos previos para conocer la distribución de las especies de Glyphis en México y el mundo; además, se construyó una clave taxonómica de las especies aquí abordadas.

Resultados clave: Glyphis scyphulifera y G. atrofusca se reportan por primera vez en el estado de Veracruz y Glyphis substriatula se cita por primera vez en México. Se incluye una clave para la determinación de las especies de Glyphis conocidas para México.

Conclusiones: De acuerdo con las colectas realizadas en la zona tropical del gradiente altitudinal de "Cofre de Perote", Veracruz, México, se encontró que cuatro de las cinco especies de Glyphis aceptadas en el mundo habitan en este lugar, estando Glyphis cicatricosa y G. scyphulifera en toda la parte muestreada del gradiente, mientras que G. atrofusca y G. substriatula están restringidas a elevaciones bajas.

Palabras clave: clave taxonómica, Graphidaceae, Graphidales, líquenes, nuevo registro.

\section{Abstract}

Background and Aims: The Graphidaceae family is considered one of the most representative of lichenized fungi, both in Mexico and in the world. Approximately 175 of its species have been recorded in Mexico. The objective of this study is to carry out a taxonomic review of Glyphis in Veracruz, through the analysis of literature and material collections from the tropical zone of the elevation gradient on the "Cofre de Perote", Veracruz, Mexico. Methods: Microlichens were collected from localities in tropical ecosystems of the altitudinal gradient of the "Cofre de Perote", Veracruz, Mexico, in cloud forest and tropical dry forest. The material was processed and deposited in the XALU herbarium and analyzed using conventional lichenology techniques for morphology and macrochemistry. Databases and previous works were consulted to know the distribution of Glyphis species in Mexico and the world; moreover, we provide a taxonomic key of the species discussed here.

Key results: Glyphis scyphulifera and G. atrofusca are reported for the first time in the state of Veracruz and G. substriatula is cited for the first time in Mexico. A key is included to determine the known Glyphis species for Mexico.

Conclusions: According to the collections carried out in the tropical zone of the altitudinal gradient of the "Cofre de Perote", Veracruz, Mexico, it was found that four of the five species of Glyphis accepted in the world inhabit this place, being Glyphis cicatricosa and G. scyphulifera throughout the sampled part of the gradient, while G. atrofusca and G. substriatula are restricted to low elevations.

Key words: Graphidaceae, Graphidales, lichens, new record, taxonomical key.

${ }^{1}$ Universidad Veracruzana, Facultad de Ciencias Bioló- Recibido: 17 de octubre de 2020.

gicas y Agropecuarias, Peñuela, 94945 Amatlán de los Revisado: 8 de enero de 2021.

Reyes, Veracruz, México.

2Universidad Veracruzana, Facultad de Biología, campus Xalapa, Circuito Gonzalo Aguirre Beltrán s/n, Zona Universitaria, 91090 Xalapa-Enríquez, Veracruz, México. ${ }^{3}$ Autor para la correspondencia: qwerty.guzmi@gmail. com
Aceptado por Marie-Stéphanie Samain: 19 de marzo de 2021.

Publicado Primero en línea: 15 de abril de 2021.

Publicado: Acta Botanica Mexicana 128(2021).
Citar como: Guzmán-Guillermo, J., C. Barrera-Bernal y R. C. Llarena-Hernández. 2021. Los hongos liquenizados del género Glyphis en Veracruz, México. Acta Botanica Mexicana 128: e1803. DOI: https://doi. org/10.21829/abm128.2021.1803 cc) (7) (8) Este es un artículo de acceso abierto (c) ${ }_{\text {EY }}$ No bajo la licencia Creative Commons 4.0 Atribución-No Comercial (CC BY-NC 4.0 Internacional). 


\section{Introducción}

En la actualidad, se está realizando un amplio debate entre los liquenólogos en relación a la naturaleza quimérica de los talos liquénicos. Estos se encuentran formados, según lo aceptado, por dos, tres o más simbiontes que le proporcionan estabilidad estructural y fisiológica a la asociación resultante (Hawksworth y Grube, 2020). Según los puntos de vista y el enfoque de los análisis, la ecología de las asociaciones liquénicas es percibida por algunos investigadores como simbiosis mutualista; mientras que para otros, como simbiosis parasitaria (Insarova y Blagoveshchenskaya, 2016). El micobionte y el fotobionte son los simbiontes protagonistas, o al menos los más evidentes. El primero corresponde a la parte fúngica (hifas) que forma el andamiaje que proporciona el medio adecuado en el cual las algas (fotobiontes) se desarrollan y producen fotoasimilados (Brodo et al., 2001). Los hongos que se pueden liquenizar incluyen, en la mayoría de los casos, miembros de Ascomycota y, en menor número, a integrantes de Basidiomycota (HerreraCampos et al., 2014). Sin embargo, las algas liquénicas pueden abarcar incluso reinos distintos; así encontramos procariotas de Cyanophyta (p. ej. Gloeocapsa Kützing y Nostoc Vaucher ex Bornet \& Flahault) y eucariotas de Chlorophyta (p. ej. Trebouxia Puymaly y Trentepohlia C. Martius) (Brodo et al., 2001).

Los talos resultantes de la asociación alga-hongo poseen formas altamente diversas y pueden ser clasificados en dos grandes grupos meramente didácticos, ya que no tienen una relación con su filogenia: los macrolíquenes y los microlíquenes. Dentro de los macrolíquenes podemos distinguir los fruticosos (p. ej., Niebla Rundel \& Bowler, Ramalina Ach., Roccella DC. y Usnea Dill. ex Adans.), foliosos (p. ej. Heterodermia Trevis, Parmotrema A. Massal, Physcia (Schreb.) Michx. y Punctelia Krong) y dimórficos ( $\mathrm{p}$. ej. Cladonia P. Browne, Dibaeis Clem., Lepidostroma Mägd. \& S. Winkl. y Phyllobaeis Kalb \& Gierl). En el caso de los microlíquenes encontramos los crustáceos (p. ej. Arthonia Ach., Glyphis Ach., Graphis Adans. y Thelotrema Ach.). Estructuralmente, estos últimos son talos compuestos por escasas capas de hifas y algas que le proporcionan un aspecto de costra o mancha en las cortezas o rocas, y que son advertidos únicamente por sus colores o sus estructuras sexuales.
De los hongos liquenizados, la familia Graphidaceae es la segunda más diversa después de Parmeliaceae; la integran líquenes crustáceos que cuentan con una amplia variedad de formas y estructuras y son la comunidad liquénica más importante en ecosistemas tropicales ( $\mathrm{Ri}$ vas Plata et al., 2013). De las conocidas para el mundo, alrededor de 175 de sus especies han sido registradas en México (Herrera-Campos et al., 2014). Recientemente, con ayuda de estudios moleculares, Graphidaceae se dividió en tres subfamilias: Fissurinoideae, Gomphilloideae y Graphidoideae (Rivas Plata et al., 2012). Esta última se dividió en las tribus Graphideae, Ocellularieae y Thelotremateae; Glyphis Ach. se ubica en Graphideae (Rivas-Plata et al., 2012).

El nombre Glyphis deriva de la palabra griega $ү \lambda \cup \phi \omega$ (glypho) que significa tallar o grabar (Archer, 2009). El género comprende especies ampliamente polimórficas cuyos ejemplares varían incluso dentro de muestras de una misma colecta (Staiger, 2002). Taxonómicamente, Glyphis fue reconocido por Acharius sin seleccionar el tipo, aunque dentro de él incluyó a Glyphis labyrinthica Ach. (= Sarcographa labyrinthica (Ach.) Müll. Arg.), Glyphis tricosa Ach. (= Sarcographa tricosa (Ach.) Müll. Arg.), Glyphis cicatricosa Ach. y Glyphis favulosa Ach. (= Glyphis cicatricosa Ach.) (Acharius, 1818). Posteriormente, algunas de estas fueron separadas de Glyphis y transferidas a Sarcographa Fée. Más tarde el concepto Glyphis fue robustecido por Clements y Shear (1931), quienes seleccionaron a G. cicatricosa como tipo del género. Recientemente, mediante análisis molecular, el concepto fue confirmado por Staiger et al. (2006) como un grupo monofilético.

Actualmente Glyphis incluye cinco especies aceptadas; sin embargo, la mayor parte de los nombres asociados a este, necesitan ser revisados debido a que representan nombres antiguos que no se han vuelto a citar, o formas y variedades taxonómicas poco conocidas. Las amplias variaciones morfológicas en el concepto Glyphis han traído consigo la existencia de un amplio número de especies y subespecies que se han venido combinando. Por ejemplo, Glyphis scyphulifera (Ach.) Staiger fue descrita dentro de Lecidea Ach., posteriormente se creó Gyrostomum Fr. como monoespecífico para acomodarla (Staiger et al., 2006). Incluso en la actualidad se han descrito hongos liqueniza- 
dos que corresponden a conceptos dentro Glyphis, como Graphis ferrugineodisca Seavey \& Seavey que corresponde a Glyphis atrofusca (Müll. Arg.) Lücking (Lücking y Kalb, 2018). En un sentido ecológico, los representantes de este género habitan ecosistemas tropicales y subtropicales, algunos como Glyphis cicatricosa son conocidos de todo el globo mientras que otros como Glyphis dictyospora Staiger solo han sido registrados en sus localidades tipo (Staiger, 2002).

El objetivo de este estudio es presentar una revisión taxonómica de Glyphis en Veracruz, la realización de este trabajo conllevó el análisis de literatura y de colectas provenientes de la zona tropical del gradiente altitudinal de Cofre de Perote (Fig. 1), Veracruz, México, el cual posee características ecosistémicas tales que le proporcionan una alta variedad de ambientes (Carvajal-Hernández y Krömer, 2015), que permitieron un estudio exhaustivo del género.

\section{Materiales y Métodos}

El material estudiado corresponde a localidades ubicadas en ecosistemas tropicales de bosque mesófilo de montaña y bosque tropical caducifolio en las zonas media y baja del gradiente altitudinal de Cofre de Perote, en los municipios Actopan, Emiliano Zapata, Plan del Rio, Tlalnelhuayocan y Xalapa, en el estado de Veracruz, México.
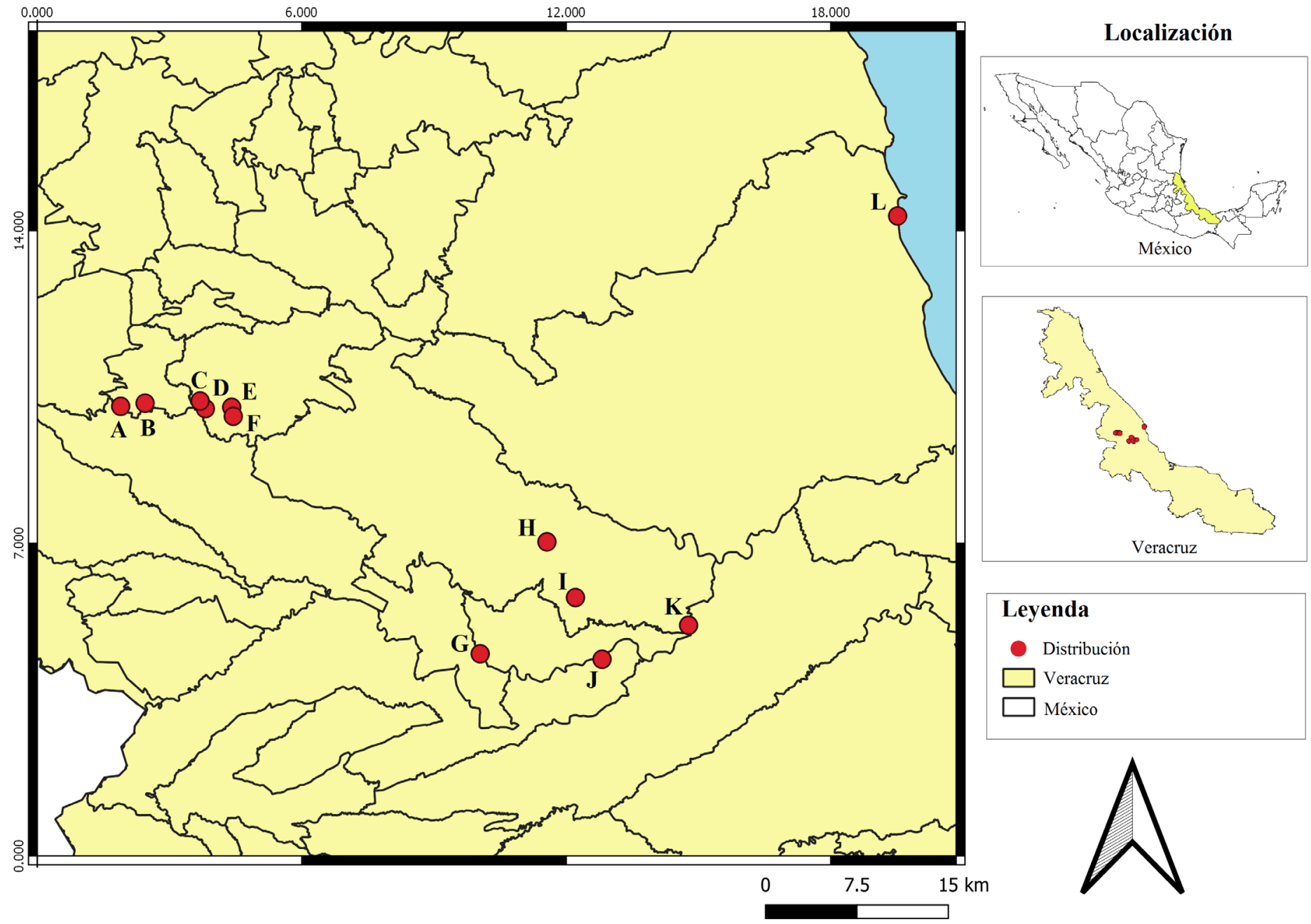

\section{Leyenda

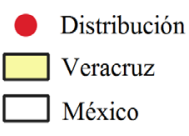

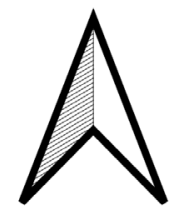

Figura 1: Ubicación de las localidades en las que se colectaron los hongos liquenizados del género Glyphis muestreados en los municipios Actopan, Emiliano Zapata, Plan del Rio, Tlalnelhuayocan y Xalapa, en el estado de Veracruz, México. Localidades: A. El Chivizcoyo; B. Los Capulines; C. Parque Ecológico El Haya; D. Santuario del Bosque de Niebla; E. Paseo de los Lagos del Dique; F. Campus para la Cultura, las Artes y el Deporte; G. Apazapan; H. La Piedra; I. sembradío de Cedrela odorata L.; J. Carrizal; K. Rinconada; L. Villa Rica de la Veracruz. 
Los ejemplares se recolectaron entre 2016 y 2020. El método de muestreo fue por conveniencia. Las muestras se procesaron bajo técnicas convencionales; se desinfectaron por enfriamiento y se depositaron en la Colección de Líquenes del Herbario XALU (acrónimos según Thiers, 2020+) de la Facultad de Biología de la Universidad Veracruzana.

Se realizaron cortes de ascocarpos a mano alzada en un microscopio estereoscópico (Carl Zeiss modelo Stemi Dv4, Jena, Alemania) y se montaron en agua, para posteriormente ser analizados mediante técnicas convencionales de liquenología ejemplificadas por Brodo et al. (2001). Sus estructuras se midieron en un microscopio (Carl Zeiss modelo Primo Star HAL/LED, Jena, Alemania) en el Laboratorio de Calidad Ambiental de la Facultad de Biología de la Universidad Veracruzana, Xalapa, México. Para conocer la macroquímica del talo se aplicaron las pruebas de punto (spot-test): C (cloro comercial), K (KOH 5\%), KC (modificación de las dos anteriores) y reacción a UV. La reacción amiloide de sus estructuras sexuales se probó con solución de Lugol (IKI) (Staiger, 2002).

La distribución previamente conocida de las especies de Glyphis se recopiló de las citas obtenidas en distintas publicaciones (Sipman y Wolf, 1998; Staiger, 2002; Archer, 2004; Staiger y Kalb, 2004; Archer, 2009; Kukwa et al., 2013; Herrera-Campos et al. 2017; Hardini et al., 2018; Luch y Lücking, 2018) y de la información que se recuperó de bases de datos en línea como Catalog of the Lichens and Lichenicolous Fungi of Mexico (Ryan et al., 1996) y el Consortium of North American Lichen Herbaria (CNALH, 2020).

\section{Resultados}

De acuerdo con los datos obtenidos de las recolectas y la bibliografía consultada, el género Glyphis está presente en el gradiente altitudinal de Cofre de Perote desde la región terrestre a nivel del mar, en la costa del Golfo de México en selva baja caducifolia, hasta los 1700 m s.n.m., en bosque mesófilo de montaña. De las cinco especies aceptadas para el género, se encontraron las siguientes cuatro: $G$. atrosfusca, G. cicatricosa, G. scyphulifera y G. substriatula (Nyl.) Staiger.

\section{Taxonomía}

\section{Ascomycota}

\section{Graphidales}

\section{Graphidaceae}

\section{Glyphis Ach., Syn. Meth. Lich. (Lund): 106. 1814.}

Glyphis se integra por especies de hongos liquenizados que forman un talo crustáceo corticado de colores variables, pudiendo ser amarillentos, verdosos o blanquecinos, poseen ascocarpos lireloides, pudiendo ser estromáticos o cuando no, de formas variables, en ocasiones poseen lirelas largas y ramificadas, cortas sin ramificaciones e incluso redondas (Staiger, 2002; Staiger et al., 2006; Lucking y Rivas-Plata, 2008), el disco himenial o abertura de los labios abundantemente cubiertos por pruina parda (Fig. 2), tienen un excípulo lateral completamente carbonizado, himenio no insperso, parafisas ramificadas apicalmente, de paredes gruesas, esporas hialinas y amiloideas pudiendo ser transversalmente septadas, muriformes o submuriformes (Lucking y RivasPlata, 2008; Staiger, 2002), el fotobionte corresponde a Trentepohlia sp. (Fig. 3), se puede diferenciar de otros géneros de Graphidaceae por poseer un disco himenial cubierto por una pruina marrón, esporas hialinas, puntas de las parafisas ramificadas y pruebas macroquímicas (C, K y P) negativas; en relación a su ecología son exclusivamente corticícolas, a excepción de Glyphis scyphulifera, que también puede desarrollarse como folíícola (Staiger, 2002). A continuación, se presenta una clave taxonómica de las especies abordadas en este trabajo y enseguida las descripciones de las especies de Gyphis encontradas en la región tropical del gradiente altitudinal de Cofre de Perote, Veracruz, México.

\section{Clave para las especies de Glyphis}

1a. Ascocarpos formando un estroma evidente y esporas transversalmente septadas ... Glyphis cicatricosa Ach.

1b. Ascocarpos solitarios y esporas muriformes ............... 2

2a. Ascocarpos redondos Glyphis scyphulifera (Ach.) Staiger 


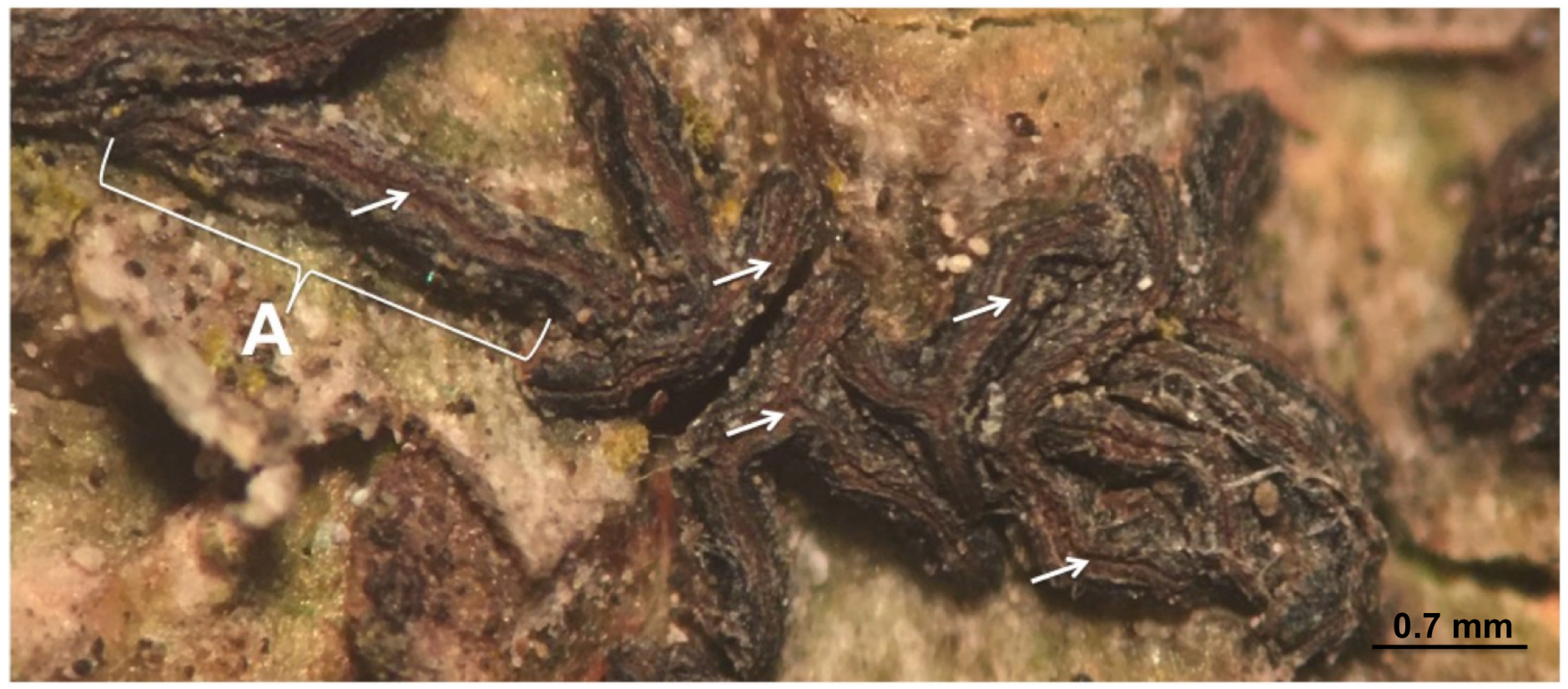

Figura 2: Lirelas (A) de Glyphis substriatula (Nyl.) Staiger, con abundante pruina parda en la abertura de los labios (flechas).

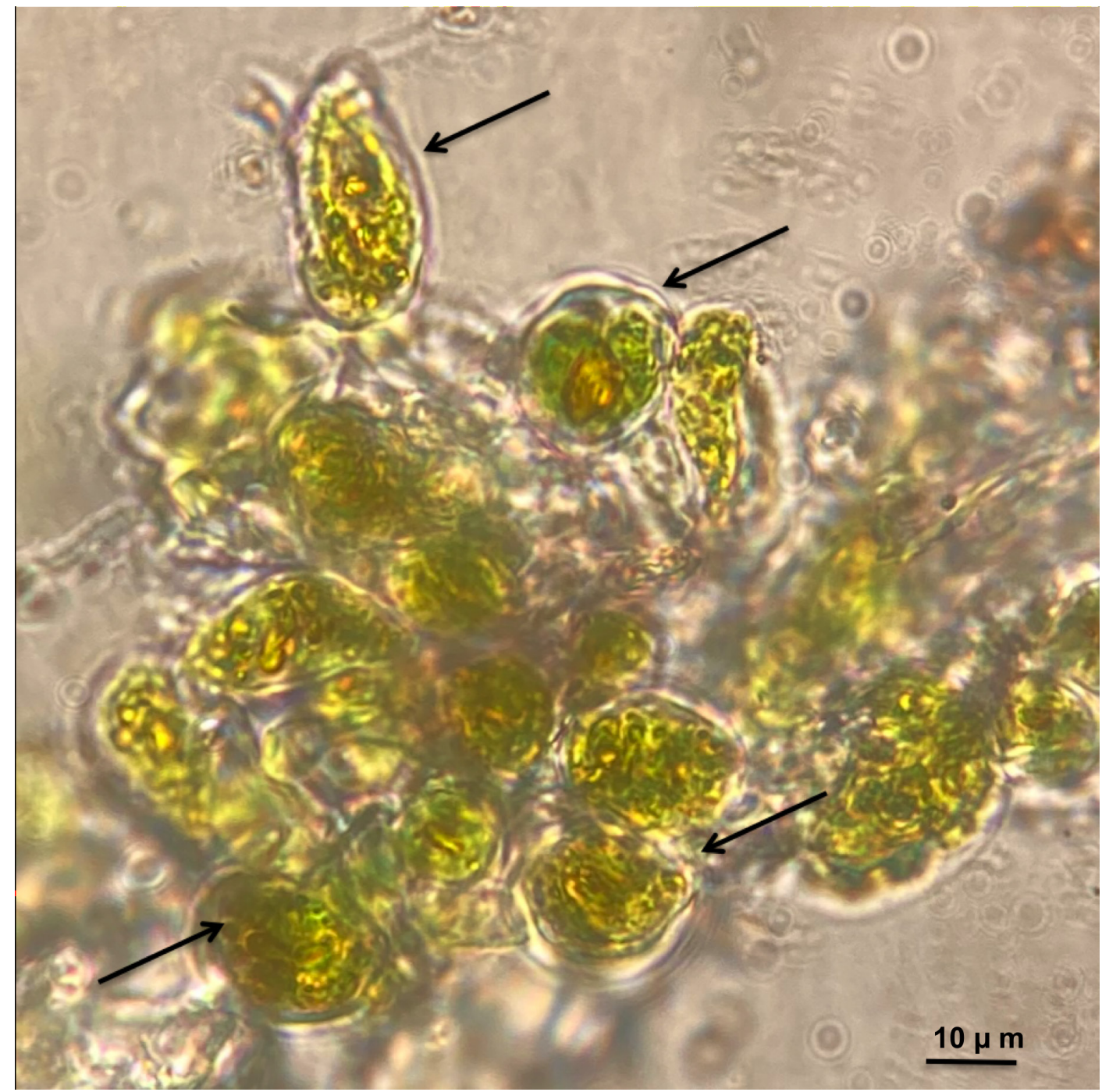

Figura 3: Algas unicelulares (flechas) del género Trentepohlia C. Martius, asociadas a hongos del género Glyphis Ach. 
2b. Ascocarpos lireliformes 3

3a. Excípulo completamente carbonizado con los labios convergentes y ascas octospóricas

Glyphis substriatula (Nyl.) Staiger

3b. Excípulo lateralmente carbonizado con los labios no convergentes y ascas bi o tetraspóricas

Glyphis atrofusca (Müll. Arg.) Lucking

Glyphis atrofusca (Müll. Arg.) Lucking, Flora of Australia, Vol. 57: 651. 2009.

TIPO: SUDÁFRICA. Mpulamalanga, Wilms 70 (lectotipo: G, designado por Lücking et al., 2009).

इ Graphina atrofusca Müll. Arg., Flora, Regensburg 70: 74. 1887.

三 Graphis atrofusca (Müll. Arg.) Stizenb., Ber. Tät. St. Gall. Naturw. Ges.: 186. 1891.

= Graphis ferrugineodisca Seavey \& J. Seavey, Bull. Florida Mus. Nat. Hist. 53(5): 225. 2017.

Figs. 4 A-C.

Talo crustáceo ecorticado, color grisaceo con coloraciones verdosas en algunas partes del talo, de aspecto continuo, no areolado; apotecios lireloides que terminan en punta o redondeadas, lirelas ramificadas o no, no estromáticos, inmersas a errumpentes, excípulo lateralmente carbonizado, disco himenial cubierto con pruina parda; ascas con 2 o 4 esporas; ascosporas hialinas, muriformes, $34-41 \times 15-20 \mu \mathrm{m}$.

Notas taxonómicas: se diferencia de otras especies de Glyphis por poseer las lirelas inmersas en el talo y tener el disco expuesto, así como la base no carbonizada (Staiger, 2002; Staiger y Kalb, 2004). Las muestras estudiadas corresponden a selva baja caducifolia entre 234 y $410 \mathrm{~m}$ s.n.m.

Reacciones: Ascos I-; esporas I+ púrpura; talo C-, K-, KC-, I-, UV-.

Sustrato: cortícola sobre Cedrela odorata L.
Distribución: pantropical. Se ha citado de América (Norte y Sudamérica), Asia y Australia (Staiger, 2002; Archer, 2009; Hardini et al., 2018). Para México se conocía previamente de Sinaloa (Staiger y Kalb, 2004).

Material estudiado: MÉXICO. Veracruz, municipio Emiliano Zapata, localidad de Carrizal, 410 m s.n.m., $19^{\circ} 24^{\prime} 40.6^{\prime \prime} \mathrm{N}, 96^{\circ} 40^{\prime 22.2 " O, ~ 29 . V I I I .2020, ~ J . ~ G u z m a ́ n-G u i-~}$ Ilermo 1820 (XALU), 1825 (XALU), 1829 (XALU), 1830 (XALU), 1831 (XALU), 1832 (XALU), 1833 (XALU), 1834 (XALU); Plan del Río, 234 m s.n.m., 19¹9'15.1"N, 96³7'47.3"O, 10.IV.2020, K.D.R. Cárdenas-Mendoza 673 (XALU), 674 (XALU).

Glyphis cicatricosa Ach., Syn. Meth. Lich.: 107. 1814.

TIPO: GUINEA. Localidad no mencionada, sobre Codarii acutifolii Afz. (holotipo: $\mathrm{H}-\mathrm{ACH} 887$ ).

= Glyphis confluens Zenker, en Goebel \& Kunze, Pharmaceutische Waarenkunde 1(3): 163. 1827.

= Glyphis cribrosa Fée, Nova Acta Phys.-Med. Acad. Caes. Leop.-Carol. Nat. Cur. Supl. 1(18): 36. 1841.

= Glyphis verrucosa C. Knight ex Shirley, Proc. R. Soc. Qd. 6: 214. 1889.

= Glyphis favulosa var. depauperata Müll. Arg., Hedwigia 30: 54. 1891.

= Glyphis cicatricosa var. confluens (Zenker) Zahlbr., Denkschr. Kaiserl. Akad. Wiss. Wien, Math.-Naturwiss. KI.

83: 112. 1927.

= Glyphis cicatricosa var. depauperata (Müll. Arg.) Zahlbr., Cat. Lich. Univers. 2: 456. 1923.

= Glyphis cicatricosa var. intermedia (Müll. Arg.) Zahlbr., Cat. Lich. Univers. 2: 456. 1923.

= Glyphis verruculosa Zahlbr., Cat. Lich. Univers. 2: 457. 1923.

Figs. 4 D-F.

Talo crustáceo ecorticado, color verdoso con coloraciones amarillentas en algunas ocasiones, de aspecto estriado, no areolado; apotecios lireloides, estromáticos, inmersos a errumpentes (varían ampliamente de acuerdo al 


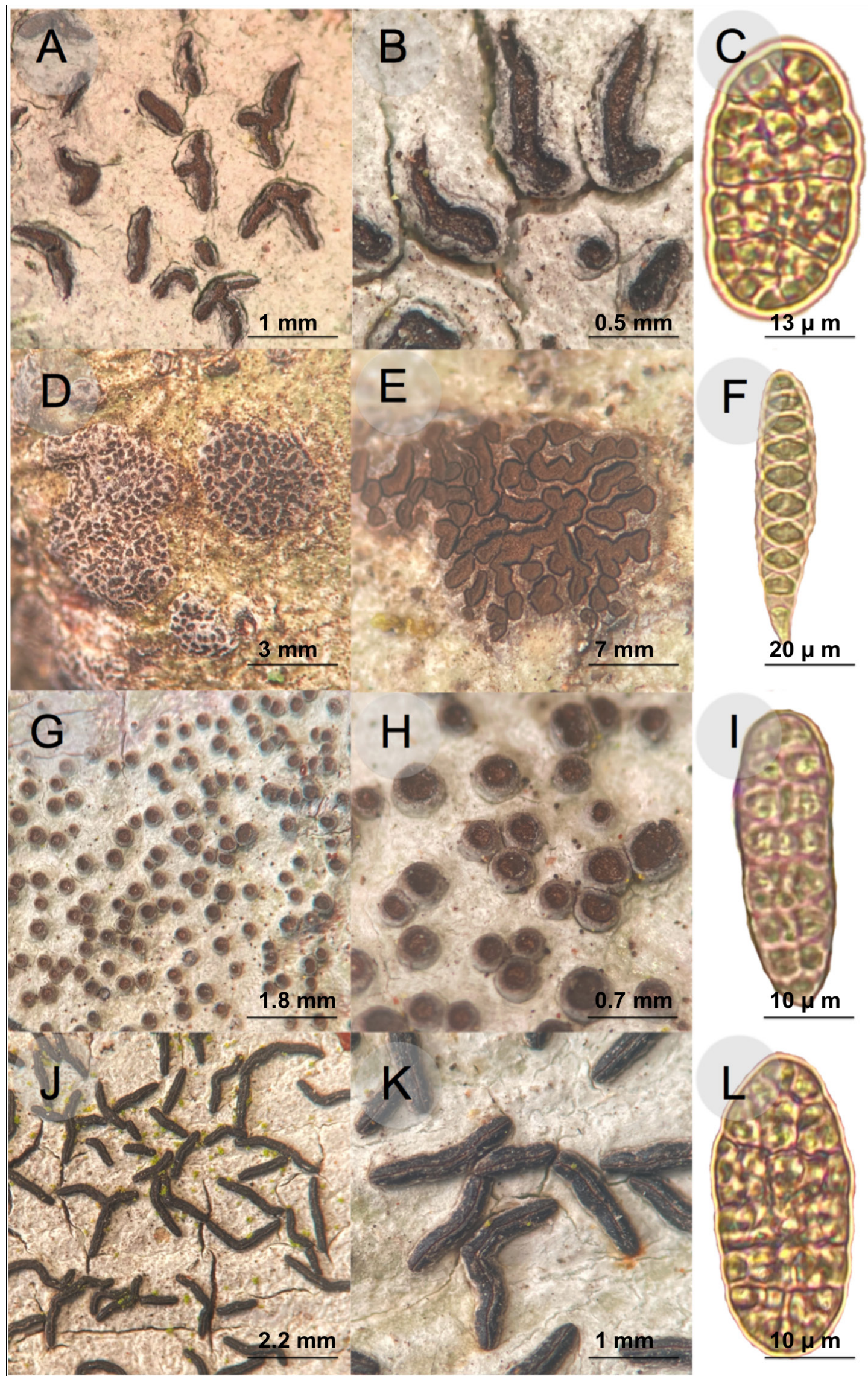

Figura 4: Especies del género Glyphis Ach. de la zona tropical del gradiente altitudinal de Cofre de Perote, Veracruz, México. A-C Glyphis atrofusca (Müll. Arg.) Lücking; D-F Glyphis cicatricosa Ach.; G-I Glyphis scyphulifera (Ach.) Staiger; J-L Glyphis substriatula (Nyl.) Staiger. 
estado de maduración), excípulo totalmente carbonizado, disco himenial cubierto con pruina parda; ascas octospóricas, clavadas, $98 \times 20 \mu \mathrm{m}$; ascosporas hialinas, fusiformes con un extremo más delgado, transversalmente distoseptadas con 9-12 lóculos, 33-40 × 8-9 $\mu$ m; arafisas ramificadas, $115 \times 1.5 \mu \mathrm{m}$.

Notas taxonómicas: otra especie de este género que posee un ascocarpo inmerso en un estroma es Glyphis dictyospora. Se logran diferenciar ya que $G$. cicatricosa tiene esporas transversalmente septadas, mientras que G. dyctiospora, muriformes (Staiger, 2002; Archer; 2004, 2009). Las muestras estudiadas corresponden a selva baja caducifolia a 234 m s.n.m. y a bosque mesófilo de montaña entre 1338 y 1605 m s.n.m.

Reacciones: Ascos I-; esporas I+ púrpura; talo K-, C-, I-.

Sustrato: cortícola sobre Liquidambar styraciflua L., Platanus mexicana Moric., Acacia sp., Quercus sp. y Cedrela odorata.

Distribución: pantropical. En México se ha encontrado en los estados de Chiapas, Hidalgo, Jalisco, Nuevo León, Puebla, San Luis Potosí y Veracruz (Ryan et al., 1996; Sipman y Wolf, 1998; Herrera-Campos et al., 2017; CNALH, 2020).

Material estudiado: MÉXICO. Veracruz, municipio Emiliano Zapata, sembradío de Cedrela odorata a $5 \mathrm{~km}$ de carretera principal en la localidad de Carrizal, 234 m s.n.m., 19¹9'15.1"N, 96³7'47.3"O, 25.II.2017, J. Guzmán-Guillermo 559 (XALU), 560 (XALU). Municipio de Tlalnelhuayocan, El Chivizcoyo, 1605 m s.n.m., $19^{\circ} 30^{\prime} 56.4942^{\prime \prime N}, 97^{\circ} 0^{\prime} 19.7814^{\prime \prime O}$, 18.VIII.2016, J. Guzmán-Guillermo 234 (XALU), 235 (XALU); Los Capulines, 23.X.2018, J. Guzmán-Guillermo 634 (XALU). Municipio Xalapa, Parque Ecológico El Haya, 1408 m s.n.m., 19³1'11.2"N, 9656'36.6"O, 19.X.2018, J. Guzmán-Guillermo 612 (XALU), 613 (XALU); loc. cit., 26.X.2018, J. GuzmánGuillermo 640 (XALU); loc. cit., 2.XI.2019, J. Guzmán-GuiIlermo 742-A (XALU); Santuario del Bosque de Niebla, 1377 m s.n.m., $19^{\circ} 30^{\prime} 50.0^{\prime \prime} \mathrm{N}, 96^{\circ} 56^{\prime} 21.9^{\prime \prime O}, 20 . X .2018$, J. Guz-
mán-Guillermo 614 (XALU); Paseo de Los Lagos del Dique, 1338 m s.n.m, 19³0'53.8"N, 9655'07.9"O, 19.X.2019, J. Guzmán-Guillermo 687 (XALU); Campus para la Cultura, las artes y el deporte de la UV, $1364 \mathrm{~m}$ s.n.m., $19^{\circ} 30^{\prime} 28.8^{\prime \prime} \mathrm{N}$, 9655'03.4"W, 20.XII.2019, J. Guzmán-Guillermo, 1315-A (XALU), 1330 (XALU), 1349 (XALU), 1359 (XALU).

Glyphis scyphulifera (Ach.) Staiger, Biblioth. Lichenol. 85: 175. 2002.

TIPO: GUINEA. Localidad no mencionada, sobre corteza (holotipo: H-ACH 286).

इ Lecidea scyphulifera Ach., Syn. Meth. Lich. (Lund): 27. 1814.

इ Gyrostomum scyphuliferum (Ach.) Nyl., Annals Sci. Nat. Bot., sér. 4(16): 96. 1867.

Figs. 4 G-I.

Talo crustáceo ecorticado, color blanquecino a ligeramente verdoso cuando húmedo; apotecios redondos, no estromáticos, sésiles, sin estípite, excípulo totalmente carbonizado y con el disco himenial cubierto con pruina parda, adicionalmente el talo puede estar cubierto por un tejido algodonoso y laxo blanco (Fig. 4E); ascas octospóricas, clavadas; ascosporas, muriformes con los extremos iguales, de 32-36 × 14-15 $\mu \mathrm{m}$, con 12-14/3-4 lóculos; parafisas ramificadas.

Notas taxonómicas: se diferencia de otras especies del género por poseer esporas muriformes, un excípulo totalmente carbonizado, ascocarpos no lireloides elongados y discoideos, además de no encontrarse embebidos en una estructura estromática (Staiger, 2002; Archer, 2009). Las muestras estudiadas corresponden a vegetación costera (región terrestre) a 0 m s.n.m, en selva baja caducifolia entre 0 y 313 m s.n.m., y a bosque mesófilo de montaña a 1408 m s.n.m.

Reacciones: ascas I-; esporas I+ púrpura; talo K-, C-, I-.

Sustrato: cortícola sobre Cedrela odorata, Spathodea campanulata Beauv., Quercus sp., Tabebuia rosea (Bertol.) 
DC., Manilkara zapota (L.) P. Royen, Liquidambar styraciflua y Platanus mexicana.

Distribución: pantropical. En México se conoce de Chiapas, Jalisco, Nuevo León y San Luis Potosí (Ryan et al., 1996; Herrera-Campos et al., 2017; CNALH, 2020).

Material estudiado: MÉXICO. Veracruz, municipio Actopan, localidad Villa Rica de la Veracruz, 0 m s.n.m., $19^{\circ} 39^{\prime} 44.2^{\prime \prime} \mathrm{N}, 96^{\circ} 23^{\prime} 56.9^{\prime \prime O}$ 8.XII.2019, J. Guzmán-Guillermo 1176 (XALU), 1179 (XALU), 1181-A (XALU), 1182 (XALU), 1183 (XALU), 1186 (XALU), 1187 (XALU). Municipio Emiliano Zapata, localidad de Apazapan, 313 m s.n.m., $19^{\circ} 19^{\prime} 30.3^{\prime \prime} \mathrm{N}, 96^{\circ} 43^{\prime 2} 29.7^{\prime} \mathrm{O}, 15.11 .2020$, J. Guzmán-Guillermo 1398 (XALU), 1424 (XALU), 1458 (XALU), 1469 (XALU), 1472 (XALU), 1476 (XALU), 1489 (XALU), 1509 (XALU); localidad Rinconada, 237 m s.n.m., 19²0'49.4' N, 96³3'44.3"O, 15.II.2020, J. Guzmán-Guillermo 1557 (XALU), 1587 (XALU); localidad Carrizal, $234 \mathrm{~m}$ s.n.m., 19¹9'15.1"N, 96³7'47.3"O, 29.VIII.2020, J. Guzmán-Guillermo 1824 (XALU), 1827 (XALU), 1829 (XALU), 1837 (XALU), 1838 (XALU). Municipio Xalapa, Parque Ecológico El Haya, 1408 m s.n.m., 19³1'11.2"N, 9656'36.6"O, 2.XI.2019, J. Guzmán-Guillermo 723 (XALU), 742-B (XALU).

Glyphis substriatula (Nyl.) Staiger Biblioth. Lichenol. 85: 175. 2002.

TIPO: NICARAGUA. Localidad no mencionada, sobre corteza, Linding 2728 (holotipo: H-NYL 7411).

E Graphis substriatula Nyl., Acta Soc. Sci. Fenn. 7(2): 467. 1863.

E Graphis elegans var. substriatula (Nyl.) Tuck., Proc. Amer. Acad. Arts \& Sci. 7: 230. 1868.

三 Graphina substriatula (Nyl.) Zahlbr., Cat. Lich. Univers. 2: 427. 1923.

Figs. 2, 4 J-L.

Talo crustáceo, ecorticado, color blanquecino a ligeramente verde amarillento cuando húmedo; apotecios lireloides, no estromáticos, sésiles, excípulo totalmente carbonizado y margen talino ausente, disco himenial no expuesto, abertura de los labios cubierta por pruina parda (Fig. 2), en ocasiones esparcida sobre toda la lirela; ascas octospóricas y de forma clavada; ascosporas muriformes con los extremos iguales, 34-38 × $11 \mu \mathrm{m}$, con 10-13/4 lóculos; parafisas ramificadas.

Notas taxonómicas: esta especie es fácilmente reconocible de otras del género Glyphis por formar lirelas sésiles con el disco no expuesto con la abertura de los lábios cubierta por pruina parda (Staiger, 2002). Se puede diferenciar de G. atrofusca ya que esta posee un excípulo lateralmente carbonizado y el disco expuesto. Las muestras estudiadas corresponden a selva baja caducifolia entre 234 y 313 m s.n.m.

Reacciones: Ascas I-; ascosporas I+ púrpura; talo C-; K-, KC-, I-.

Sustrato: cortícola sobre Cedrela odorata.

Distribución: se conoce de Centroamérica, Sudamerica (Staiger, 2002; Cáceres, 2007; Kukwa et al., 2013), Hawaii (Luch y Lücking, 2018) y Asia (Hardini et al., 2018). El material aquí estudiado corresponde al primer registro de la especie en México.

Material estudiado: MÉXICO. Veracruz, municipio Emiliano Zapata, localidad Apazapan, 313 m s.n.m., $19^{\circ} 19^{\prime} 30.3^{\prime \prime} \mathrm{N}, 96^{\circ} 43^{\prime 29.7 " O, ~ 15 . I I .2020, ~ J . ~ G u z m a ́ n-G u i-~}$ llermo 1468 (XALU), 1487 (XALU); localidad Carrizal, 234 m s.n.m., 19¹9'15.1'N, 96³7'47.3"O, 29.VIII.2020, J. Guzmán-Guillermo 1819 (XALU), 1821 (XALU), 1822 (XALU), 1823 (XALU), 1826 (XALU), 1828 (XALU); localidad Rinconada, 237 m s.n.m., 19²0'49.4"N, 96³3'44.3"O, 15.II.2020, J. Guzmán-Guillermo 1566 (XALU), 1581 (XALU).

\section{Discusión}

La distribución, química y características morfológicas macroscópicas y microscópicas de los ejemplares aquí estudiados coinciden con lo reportado para estas especies en otras regiones (Staiger, 2002; Archer, 2004, 2009). Si bien en el presente trabajo se registran cuatro de las cinco entidades específicas de Glyphis aceptadas, son nece- 
sarios trabajos moleculares que corroboren el concepto de Glyphis dictyospora Staiger separado de G. cicatricosa, pues no existe ninguna referencia de la primera más que su descripción original en Staiger (2002). Es necesaria la revisión de Glyphis batuana Hardini, Kasiamdari \& Purnomo, ya que de acuerdo con las características de la descripción que proveen Hardini et al. (2018), el primer autor de este trabajo considera que podría tratarse de alguna especie previamente descrita dentro de Glyphis o Graphis. Para este análisis, al igual que en el primer caso, es necesario corroborar mediante estudios moleculares la validez y posición taxonómica de esos nombres y la revisión del material tipo, ya que las fotografías e ilustraciones provistas por Hardini et al. (2018) y Staiger (2002) respectivamente, no permiten concluir el análisis sobre los conceptos mencionados.

Del material estudiado se observó que la abundancia de G. cicatricosa aumenta en ecosistemas húmedos arriba de 1000 m s.n.m., especialmente en bosque mesófilo de montaña; mientras que, $G$. scyphulifera prospera en los secos, a menos de 1000 m s.n.m. En el caso de Glyphis atrofusca y $G$. substriatula, estas solo fueron recolectadas en selva baja caducifolia a menos de 500 m s.n.m. Sin embargo, se requieren estudios ecológicos que consideren datos climáticos, para conocer la distribución de Glyphis en relación con variables climáticas, pues para otras regiones del mundo (Asia) se han asociado algunas de sus especies a climas calientes y alturas cercanas al mar (Ohmura y Seaward, 2017).

Staiger y Kalb (2004) mencionan la presencia de $G$. montoensis, cuyo nombre correcto es G. atrofusca (Lücking et al., 2009), para las costas de Sinaloa. En este trabajo, esta se cita por segunda ocasión para México, lo cual ubica a Veracruz como el estado con mayor número de taxones específicos de Glyphis, al poseer todas los representantes del género que se conocen de México. Esto se relaciona con la variedad de ambientes que se pueden encontrar en el gradiente altitudinal de Cofre de Perote.

\section{Conclusiones}

De las especies estudiadas, $G$. substriatula es un primer registro para México y G. scyphulifera y G. atrofusca son nuevos registros para Veracruz. En total, para este estado se reconocen cuatro especies: G. atrofusca, G. cicatricosa, $G$. scyphulifera y $G$. substriatula. Es necesario continuar con los estudios monográficos de los microlíquenes de Veracruz, así como de la generación de información taxonómica detallada donde se aborden los microlíquenes del sur de México, una región liquenológicamente poco explorada.

\section{Contribución de autores}

JGG diseñó el estudio con apoyo de CBB y RLH. JGG realizó las descripciones morfológicas, RLH y JGG adquirieron la información bibliográfica y su interpretación para la identificación taxonómica y JGG escribió el manuscrito con la ayuda de CBB y RLH. Todos los autores participaron a la discusión, revisión y aprobación del manuscrito final.

\section{Financiamiento}

Esta investigación se financió con recursos propios.

\section{Agradecimientos}

Se agradece al Laboratorio de Calidad Ambiental de la Facultad de Biología campus Xalapa y a los profesores F. Ortega y R. Ortega del Herbario XALU de la Universidad Veracruzana, por las atenciones brindadas durante la realización de esta investigación.

\section{Literatura citada}

Acharius, E. 1818. VII. Glyphis and Chiodecton, two new Genera of the Family of Lichenes, with Descriptions and Figures of the Species hitherto discovered. Transactions of the Linnean Society of London 12(1): 35-47. DOI: https://doi. org/10.1111/j.1095-8339.1817.tb00219.x

Archer, A. W. 2004. The lichen genera Cyclographina, Diplogramma, Glyphis, Gymnographa, Medusulina, Sarcographa and Sarcographina (Graphidaceae) in Australia. Telopea 10(2): 589-605.

Archer, A. W. 2009. Glyphis. Flora of Australia 57: 84-194.

Brodo, I. M., S. D. Sharnoff y S. Sharnoff. 2001. The Lichens of North America. Yale University Press. New Haven, USA. 795 pp.

Cáceres, M. E. S. 2007. Corticolous crustose and microfoliose lichens of notheastern Brazil. Libri Botanici 22: 1-168. 
Carvajal-Hernández, C. I. y T. Krömer. 2015. Riqueza y distribución de helechos y licófitos en el gradiente altitudinal del Cofre de Perote, centro de Veracruz, México. Botanical Sciences 93(3): 601-614. DOI: https://doi.org/10.17129/botsci.165

Clements, F. E. y C. L. Shear. 1931. The genera of fungi. The H.W. Wilson Company. New York, USA. 498 pp.

CNALH. 2020. Consortium of North American Lichen Herbaria. National Science Foundation. Alexandria, USA. https:// lichenportal.org/cnalh/collections (consultado enero de 2020).

Hardini, J., R. S. Kasiamdari, S. Santosa y P. Purnomo. 2018. A new species of Glyphis Ach. and three new records of Graphis Adans. (Graphidaceae) from Bali Island, Indonesia. Bangladesh Journal of Plant Taxonomy 25(2): 159-166. DOI: https://doi.org/10.3329/bjpt.v25i2.39520

Hawksworth, D. L. y M. Grube. 2020. Lichens redefined as complex ecosystems. The New Phytologist 227(5): 1281-1283. DOI: https://doi.org/10.1111/nph.16630

Herrera-Campos, M. A., R. Lücking, R. E. Pérez-Pérez, R. MirandaGonzález, N. Sánchez, A. Barcenas-Peña y T. H. Nash. 2014. Biodiversidad de líquenes en México. Revista Mexicana de Biodiversidad 85(Supl. 1): 82-99. DOI: https://doi. org/10.7550/rmb.37003

Herrera-Campos, M. A., R. Miranda-González, R. Lücking, N. Sánchez-Téllez y A. Barcenas-Peña. 2017. Inventario y base de datos de los líquenes de la selva seca de Jalisco, Informe final Sistema Nacional de Información sobre Biodiversidad. Comisión Nacional para el Conocimiento y Uso de la Biodiversidad, proyecto No. JF157. Instituto de Biología, Universidad Nacional Autónoma de México. Cd. Mx., México. https://www.gbif.org/es/dataset/34ca940ffacb-4039-9ac0-111dad7fd9cc (consultado enero de 2020).

Insarova, I. D. y E. Y. Blagoveshchenskaya. 2016. Lichen symbiosis: Search and recognition of partners. Biology Bulletin 43(5): 408-418.

Kukwa, M., U. Schiefelbein y A. Flakus. 2013. A Contribution to the Lichen Family Graphidaceae (Ostropales, Ascomycota) of Bolivia. Herzogia 26(2): 231-252. DOI: https://doi. org/10.13158/heia.26.2.2013.231

Luch, R. M. y R. Lücking. 2018. The genus Halegrapha new to Hawaii, with the new and potentially endemic species $H$. paulseniana and an updated checklist of Hawaiian lirellate Graphidaceae (Ascomycota: Ostropales). Willdenowia 48(3): 415-423. DOI: https://doi.org/10.3372/wi.48.48311

Lücking, R. y E. Rivas-Plata. 2008. Clave y guía ilustrada para géneros de Graphidaceae. Glallia 1(1): 1-39.

Lücking, R. y K. Kalb. 2018. Formal Instatement of Allographa (Graphidaceae): How to Deal with a Hyperdiverse Genus Complex with Cryptic Differentiation and Paucity of Molecular Data. Herzogia 31(p1): 535-561. DOI: https://doi. org/10.13158/heia.31.1.2018.535

Lücking, R., A. W. Archer y A. Aptroot. 2009. A world-wide key to the genus Graphis (Ostropales: Graphidaceae). The Lichenologist, 41(4): 363-452. DOI: https://doi.org/10.1017/ S0024282909008305

Ohmura, Y. y M. R. D. Seaward. 2017. Is Glyphis cicatricosa an indicator for "global warming" or an "urban heat island" effect in Japan? Lichenologist 49(3): 291-296. DOI: https:// doi.org/10.1017/S0024282917000111

Rivas-Plata, E., R. Lücking y H. T. Lumbsch. 2012. A new classification for the family Graphidaceae (Ascomycota: Lecanoromycetes: Ostropales). Fungal Diversity 52(1): 107121. DOI: https://doi.org/10.1007/s13225-011-0135-8

Rivas-Plata, E., S. Parnmen, B. Staiger, A. Mangold, A. Frisch, G. Weerakoon, J. E. Hernández M., M. E. S. Cáceres, K. Kalb, H. J. M. Sipman, R. S. Common, M. P. Nelsen, R. Lücking y T. Lumbsch. 2013. A molecular phylogeny of Graphidaceae (Ascomycota, Lecanoromycetes, Ostropales) including 428 species. MycoKeys 6: 55-94. DOI: https://doi.org/10.3897/ mycokeys.6.3482

Ryan, B. D., T. H. Nash III y M. A. Herrera-Campos. 1996. Catalog of the Lichens and Lichenicolous Fungi of Mexico. https:// n9.cl/gt4m (consultado enero de 2020).

Sipman, H. J. M. y J. H. D. Wolf. 1998. Provisional checklist for the lichens of Chiapas. Acta Botanica Mexicana 45: 1-29. DOI: https://doi.org/10.21829/abm45.1998.808

Staiger, B. 2002. Die flechtenfamilie Graphidaceae. Studien in Richtung einer natürlichen Gliederung. Bibliotheca Lichenologica 85: 1-526.

Staiger, B. y K. Kalb. 2004. Glyphis. In: Nash III, E. T. H., B. D. Ryan, P. Diederich, C. Gries y F. Burgartz (eds.). Lichen Flora of the Greater Sonoran Desert Region, Vol. 2. Lichens Unlimited. Arizona State University. Tempe, USA. Pp. 117-118. 
Staiger, B., K. Kalb y M. Grube. 2006. Phylogeny and phenotypic variation in the lichen family Graphidaceae (Ostropomycetidae, Ascomycota). Mycological Research 110(7): 765-772. DOI: https://doi.org/10.1016/j. mycres.2006.05.003
Thiers, B. 2020+. Index Herbariorum. A global directory of public herbaria and associated staff. New York Botanical Garden's Virtual Herbarium. http://sweetgum.nybg.org/science/ih (consultado enero de 2020). 